\title{
Machine-to-machine communications energy efficiencies: the implications of different M2M communications specifications
}

\section{Sylvester Ajah*, Ali Al-Sherbaz, Scott Turner and Phil Picton}

\author{
Department of Computing, \\ School of Science and Technology, \\ University of Northampton, \\ St Georges Ave, Northampton NN2 6JD, UK \\ Email: sylvester.ajah@northampton.ac.uk \\ Email: ali.al-sherbaz@northampton.ac.uk \\ Email: scott.turner@northampton.ac.uk \\ Email: phil.picton@northampton.ac.uk \\ *Corresponding author
}

\begin{abstract}
The energy inefficiency of electronic devices is the major limiting factor for the adoption of these devices in Machine-to-Machine Communications (M2M)/Internet of Things (IoT). In order to address this, different emerging M2M communications standardisation organisations have proposed perceived energy efficient protocols. The essence of this paper is to discuss the energy implications of different M2M communication standards on the lifespan of the electronic devices that are to be used for the M2M communications.
\end{abstract}

Keywords: M2M; machine-to-machine communications; energy efficiency; M2M standards; IoT; Internet of Things; SDO; standard development organisations.

Reference to this paper should be made as follows: Ajah, S., Al-Sherbaz, A., Turner, S. and Picton, P. (XXXX) 'Machine-to-machine communications energy efficiencies: the implications of different M2M communications specifications', Int. J. Wireless and Mobile Computing, Vol. X, No. Y, pp.xxx-Xxx.

Biographical notes: Sylvester Ajah is a PhD Student in the University of Northampton, UK. His first degree was on Electrical/Electronic Engineering at Enugu State University of Science and Technology (ESUT), Nigeria, in 2008. He did his MSc in Wireless Systems at University of Bath in 2012. His research focus is on the energy efficiency of machine to machine (M2M) communications devices, and he has one conference publication title 'Sub 1GHz M2M Communications Standardization: The Advancement in White Space Utilization for Enhancing the Energy Efficiency'.

Ali Al-Sherbaz is currently a Senior Lecturer in School of Science and Technology and skilled Research Engineer with a PhD in Wireless Communications and Network Security, and MSc in Electronic and Communication Engineering and has 20 years of theoretical and practical experience in researching and teaching at various universities both in the Middle East and in the UK, with good experience in both academic and industrial projects. He has membership with the BCS British Computer Society, IEEE (Institute of Electrical and Electronic Engineering), IEEE Communication Society, IEEE Standards Associations and Member in the Institution Engineering and Technology (IET).

Scott Turner, Associate Professor in the Department of Computing and Immersive Technologies, University of Northampton, has PhD (University of Leicester) in Neurophysiological Signal Processing and Genetic Algorithms, MSc (University of Dundee) in Biomedical Instrumentation Engineering and BEng (University of Hull) in Electronic Engineering. His research interests include robotics, problem-solving, genetic algorithms, neural networks and signal processing.

Phil Picton did his degree in Electrical and Electronic Engineering at Swansea University (1979), and PhD in Digital Logic Design at Bath University (1982). He was a Research Fellow at HeriotWatt University in Edinburgh and later a Lecturer in Electronics at the Open University. In 1994 he moved to Northampton University where he became Professor of Intelligent Computer Systems. In 2005 he became Head of Engineering, and began working closely with the British Institute of Nondestructive Testing, to produce a unique set of distance learning courses in NDT. Over his academic career he has published over a 100 papers and written two books. 


\section{Introduction}

Machine-to-Machine Communications (M2M) is a new paradigm that enables electronic devices to independently execute a task with or without human interventions. According to Ericsson (2011) there will be more than 50 billion connected devices by the end of this decade. But the major bottleneck militating against M2M communications among other challenges is the energy inefficiency of the electronic devices that are expected to be used for M2M communications. This is partly as a result of the stagnant rate of battery technology development; Wu et al. (2013) observed that it takes approximately 35 years to double the battery capacity of mobile devices. And on the energy inefficient algorithms that are used for today's wireless communications; Chen et al. (2011) noted that the design of the present common wireless network focuses on access and capacity; hence, the need to shift focus to energy efficient design. Also, Guowang et al. (2009) observed that the battery technology has not progressed as fast as the semiconductor technology; hence the energy efficiency of the wireless devices needs a cross layer approach to actualise (Miao et al.,2009).

For better comprehension of this work, it is useful to differentiate between commonly confusing used terms in M2M communications, which are: Wireless Sensor Networks (WSN), (M2M) and Internet of Things (IoT). WSN is the collection of spatially distributed wireless sensors for monitoring the physical conditions, like noise, temperature, pressure, and transmit the collective data through the wireless network to a server which stores and processes the data. The wireless connections between the nodes are through Short-Range Radio (SRR) services; and Kim and Choi (2010) observed that WSN technology does not consider the global identity for each sensor node or the location management schemes for routing because of its locality nature. M2M refers to the technologies that allow autonomous communications between similar electronic devices which can be either wired or wireless. M2M technology can provide the global view of the devices and hence can be used to interconnect the scattered WSNs (Kim and Choi, 2010). Also, Wan et al. (2013) observed that WSN and $\mathrm{M} 2 \mathrm{M}$ are similar in numerous networking aspects, but there are major differences in architecture, design and philosophy. While WSNs are designed to deliver sensor-related data, M2M enables autonomous communications between M2M devices with limited or no human intervention. Finally, IoT can be described as the connection of all the electronic devices around us in internet-like structure. The devices have global identity like IP (internet protocol addresses) which can be used to communicate them directly. In addition, M2M has been described as B2B (Business-to-Business) point solutions in which the remote devices will communicate to the central server via the network connection (internet) and the server processed data will be distributed via the internet to the devices; while IoT is B2B2C (business-to-business-toconsumer) (Beecham Research, 2012). This implies that WSN is a form of $\mathrm{M} 2 \mathrm{M}$ and $\mathrm{M} 2 \mathrm{M}$ is a form of IoT, as shown in Figure 1.
This paper evaluates different efforts by different standardisation organisations and the impact of such efforts on the lifespan of the M2M communication devices. This paper is first of their kind that exclusively discusses the works of many SDOs and the techniques they applied in enhancing the energy efficiency of the devices. This paper is organised as follows: In Section 2, we present the M2M standardisation activities by the major SDOs; followed by the M2M architecture in Section 3, the energy implications of different standardisation activities on the lifespan of the M2M devices are presented in Section 4, and finally, the open research topics and the conclusion are presented in Sections 5 and 6 , respectively.

Figure 1 The relationship between WSN, M2M and IoT (see online version for colours)

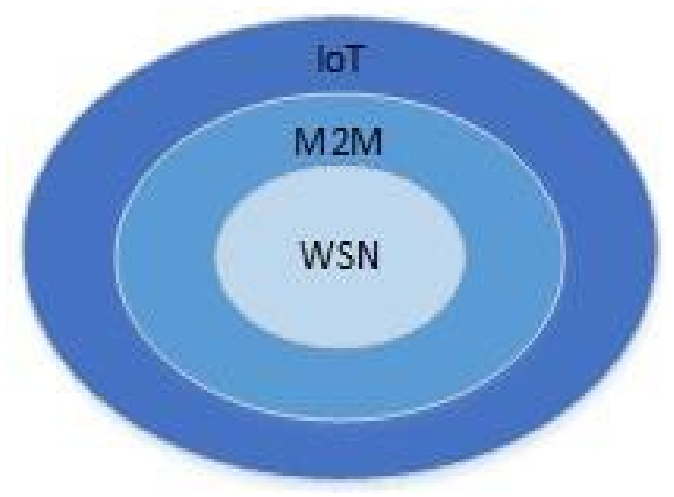

\section{M2M standardisation}

The concept of M2M standardisation became popular towards the end of last decade, because of the need to enable interconnections between different vertical M2M applications that were deployed by various communication companies and the need to have a common M2M architecture on which the existing and new direct/indirect M2M companies could develop their products. GSC (2013) observed various efforts that are being made by different world SDOs in bringing out common $\mathrm{M} 2 \mathrm{M}$ standards that will enable rapid $\mathrm{M} 2 \mathrm{M}$ developments, convergence of the already existing M2M applications and remove heterogeneity, which will catalyse M2M communications into a horizontal communications paradigm (Kim et al., 2013). The M2M ecosystem will include all the M2M stakeholders: M2M device manufacturers, ISPs (Internet Service Providers or Network providers (NPs)), platform providers, service providers and service users. As a consequence, it is expected that there will be a huge expansion in the applications of M2M communication devices, as shown in Figure 2, which will result in huge incomes to M2M companies. Following this, the GSC (2013) stated that over 143 organisations are working assiduously for direct/indirect standardisation of the M2M/IoT paradigm. In view of the above, the following sub-sections will discuss some of the major M2M standardisation activities. 


\subsection{Weightless Special Interest Group (SIG)}

The Weightless Special Interest Group (SIG) is a standardisation organisation formed in 2011 to use white space (unused TV channel - 470-790 MHz) as a medium for M2M communications, whose aim is to form an M2M standard called the weightless standard. The weightless standard is a global open standard for the IoT with a primary objective of low-cost connectivity for all the anticipated M2M applications. The proposed weightless standard is expected to have the following features: support a large number of M2M communications terminals, M2M devices long battery life, cheap M2M communications equipment and service, mobility of M2M communication devices; long range, low signal attenuation, and efficient indoor and outdoor connections (Webb, 2012). The latest weightless M2M communication specifications called weightless core specification v1.0 was published in 2013 (Weightless, 2013) which aimed at cheap energy efficient horizontal M2M applications using white space.

\subsection{DASH 7 alliance}

This group is a wireless data communications technology that was formed to enable RFID-like identification for item management and sensing systems. It is a non-profit industry conglomerate, formed in 2009 to promote the use of ISO 1800-7 (radio frequency identification for item management) standards for wireless sensor network (Mohammad et al.,
2012; Maarten et al., 2013; Sam, 2010). The body uses a $433 \mathrm{MHz}$ spectrum for its communication, which gives it the low-energy consumption, long-range communications, low cost of infrastructure deployments, low-signal attenuation, and efficient indoor and outdoor connections, advantages over some other M2M SDOs. The applications are mainly in the following: consumer electronics, pharmaceuticals, energy, home automation, agriculture, aeronautical and automotive (DASH7 Alliance, 2011; Usman and Shami, 2012).

\subsection{Zigbee alliance}

ZigBee formed in 2002 is a low-cost wireless mesh network standard and can be said to be one of the foremost groups whose works are centred on M2M/IoT communications technology (Hersent et al., 2011). The ZigBee standard is based on the IEEE 802.15.4 standard, which uses $2.4 \mathrm{GHz}$, $915 \mathrm{MHz}$ and $868 \mathrm{MHz}$ ISM bands for communications (National Instruments, 2013). The low-energy consumption, long communications range, secure networks, cheap and low throughput characteristics of ZigBee made it one of the popular M2M/IoT standardisation activities when compared with other existing standards like Wi-Fi and Bluetooth. The applications of the ZigBee standard include smart homes, commercial building automation, consumer electronics, ehealth, retail management, smart--grid, etc. (ZigBee Alliance, 2012; ZigBee, 2010; ZigBee Alliance, 2010).

Figure 2 The anticipated M2M applications (Beecham Research Limited, 2009) (see online version for colours)

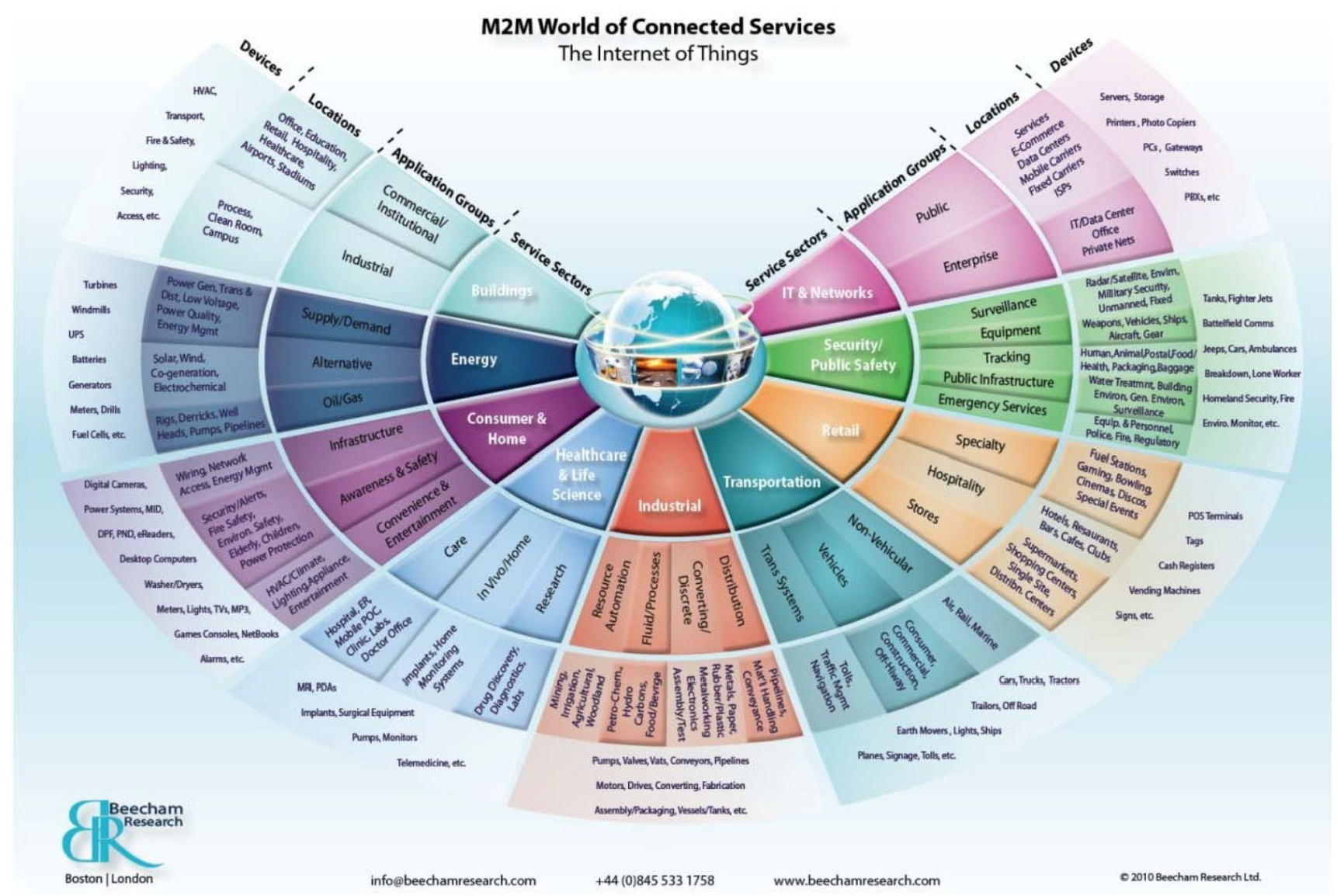




\subsection{IETF task group on M2M/IoT}

The Internet Engineering Task Force (IETF) is one of the world renowned internet connectivity experts, who formed their first task force on M2M/IoT applications called 6LoWPAN (IPv6 over low-power wireless personal area network) in 2004, with their first technical specification in 2007 (RFC 4919) (Hersent et al., 2011; 6LowPAN, 2014; 6LowPAN, 2014). 6LoWPAN was established to enable the running of IP over low power and lossy networks or batterypowered networks that were used for home and industrial automation. This was followed by another working group called IETF (Routing Over Low-power and Lossy networks - ROLL) in 2008 to create an IP level routing protocol adapted to the requirement of mesh networking for IoT with their first specification called RPL (Routing Protocol for Low-power and Lossy networks) in 2011 (Levis et al., 2009; Ko et al., 2011; Hersent et al., 2011).

\subsection{ETSI TC M2M}

European Telecommunications Standards Institute Technical Committee on M2M hope to provide an end-to-end M2M standard which they hope to achieve by working closely with ETSI's works on the next generation networks and with 3GPP standards and standardisation activities for mobile communications technologies. Their earlier work on M2M standardisation was their published technical report (use case) for smart metering (ETSI, 2011a), technical report (use case) for e-health (ETSI, 2009), technical report on automotive applications (ETSI, 2013a), technical report use cases for M2M applications for connected consumer (ETSI, 2013b) and other M2M applications technical report initiatives. Their technical specifications cover M2M service requirements, interfaces and architectures for the M2M applications they are working on, with their connection medium being the existing wireless network standards which will be mainly a combined connectivity of cellular networks (GSM, UMTS, LTE, LTE-advanced) and short-range radio networks (IEEE 802.11 standards) (ETSI, 2011b). Their main standardisation objective is to provide a service-oriented end-to-end M2M standard, though without underpinning the specific spectra or technologies for communications. Based on their proposed M2M structure, M2M devices can either connect to the network domain directly or through the gateways (Anthony et al., 2013).

\section{$2.6 \quad I T U-T$}

The International Telecommunications Union is a telecommunications standardisation advisory group (ITUTSAG) that established a technical committee called the global standards initiative on Internet of Things (IoT-GSI) to promote a consolidated path in ITU-T for developing a technical standard that will enable the IoT in the global scale in 2011 (ITU [182], 2011). To achieve this aim, they adjusted ITU-T questions from the study groups on IoT, which acts as a guideline for developing the standard and they hope to unify IoT development worldwide by working in collaboration with other SDOs.

\subsection{IEEE 802.16'S M2M TG}

The Institute of Electrical and Electronics Engineers (IEEE) is one of the world's standardisation organisations that established an M2M task group from their wireless broadband standards for local metropolitan area networks called the IEEE 802.16's M2M task group to develop, amend and enhance the air interface for broadband wireless access system for a metropolitan area network standard to support M2M communications applications in 2011 (IEEE Standards Association, 2012a). This standard is based on IMT 2000 standard around the radio spectrum of $2000 \mathrm{MHz}$. The aim of the group is to enable a range of M2M applications in Wireless Wide Area Network (WWAN) communications in the licensed bands. The IEEE WIMAX Forum, which also working of the M2M standardisation is also a subsidiary of this group (WIMAX Forum, 2014). The group has an interest in the following M2M applications: surveillance, tracking, vehicular telematics, e-health, smart metering, etc.

\subsection{Other IEEE standards}

The IEEE 802.15.4 standard which is a wireless Medium Access Control (MAC) and physical layer (PHY) specification for Low-Rate Wireless Personal Area Networks (LR-WPANs) was established in 2003, and has being used widely on M2M/IoT because of its inherent features. They also established a task group for the amendment of IEEE 802.15.4 standard that support the PHY for active RFID system bi-directional and location determination applications in 2006 using 433 ISM bands with their first specification in 2011 (IEEE Standards Association, 2012b).

\section{$2.93 G P P S 1$ and $3 G P P 2$}

The 3rd generation partnership project stage 1 (3GPP S1) protocol technical specification group is one of the pioneers of M2M standardisation working groups set up by 3GPP to optimise the Long-Time Evolution Advance (LTE-A) interfaces for M2M communications. The group brought out their first specification for M2M communications called 3GPP TS 22.368 V10.1.0 in June 2010 (3GPP, 2010) and have provided so many optimised versions of the same specification afterwards (3GPP, 2011; ETSI, 2011; 3GPP, 2012; 3GPP, 2013). In addition, the 3 rd generation partnership project two (3GPP2) technical specification groups that was also set up by 3GPP to optimise CDMA-2000 air interfaces for M2M communications, the group also brought out their technical specification for M2M communications in December 2010 called S.R0141-0 V1.0 (3GPP2, 2010). The specifications aimed at horizontal M2M applications using cellular network (CDMA-2000 and LTE-advanced). 


\section{M2M architecture}

In line with ensuring energy efficiency of M2M devices, different SDOs have proposed different architectures as the means of actualising this dream, the objective of this section is to evaluate different M2M structures being proposed by different SDOs, with the view of understanding the energy cost of them. The sub-sections below discuss the major architectures that could be used for M2M communications.

\subsection{Clustered-based M2M structure}

A cluster-based M2M structure is one of the most popular $\mathrm{M} 2 \mathrm{M}$ architects that have wide application in M2M communications especially in WSN. This is as results of the following: energy saving, network scalability and network topology stability that characterise the cluster structure. The clustering structure is necessitated by spectrum scarcity and energy efficiency associated with short-range communications. The cluster-based M2M algorithms can largely be grouped into three sub-groups as discussed in more detailed in the following sub-sections.

\subsubsection{Static WSN structure with Channel Heads (CH)}

This could be said to be the most popular WSN structure in which the sensor nodes transmit to their $\mathrm{CHs}$ which fuse the node's data together and transmit the data onwards to the sink. Heinzelman et al. (2002) proposed a Low-Energy Adaptive Clustering Hierarchy (LEACH), which will rotate the cluster heads randomly among the cluster members, which then communicate with the sink to balance the energy among the sensors. The randomness in the selection of the $\mathrm{CH}$ was believed to be incongruous because of the chance of re-selection of the earlier $\mathrm{CHs}$ as the new ones with the associated latency and energy usage of the nodes in the network coupled with the energy cost of the long-range communications associated with some $\mathrm{CH}$ and non-optimal distributions of the CHs (Muruganathan et al., 2005). Also, LEACH-C was proposed by $\mathrm{Wu}$ and Wang (2010) in which the BS uses the location and the energy level information of the nodes to form the clusters with more centred $\mathrm{CH}$ to minimise energy loss via attenuation. In another method, Muruganathan et al. (2005) propose a centralised energy efficient routing protocol called Base-Station Controlled Dynamic Clustering Protocol (BCDCP) in which the BS will set up the clusters- $\mathrm{CH}$ based on their location and the routing path to the sink which could incorporate $\mathrm{CH}$-to- $\mathrm{CH}$ routing to send the data to the sink, carry out rotation of the $\mathrm{CH}$ among the cluster members and ensures balance and uniform of the CHs in WSN field.

\subsubsection{Multi-hop-based WSN structure}

This type of WSN-based structure is necessitated by the energy efficiency associated with short-distance communications. In this structure, a node will transmit data to the next node which is on its route to the sink. PEGANESIS was proposed by Lindsey and Raghavendra (2002) in which each sensor uses the WSN, transmitting the sensed data to the nearest node towards the BS, which will fuse the data into their sensed data and send to the next node till the last node that is closest to the BS will fuse all the data together before transmitting to BS. In a similar manner, Tsa et al. (2012) proposed a node data aggregation in which the node(s) will transmit their sensed data to the next node on their routing path to the BS, the later node will buffer the data for a predetermined time before transmitting to the next node until the data gets to the gateway, which then transmits it to the BS. This is because of the ability of the M2M device to retrieve sensed data from similar M2M devices. This does improve the energy efficiency when compared to the static WSN.

Figure 3 Static WSN structure with CH (see online version for colours)

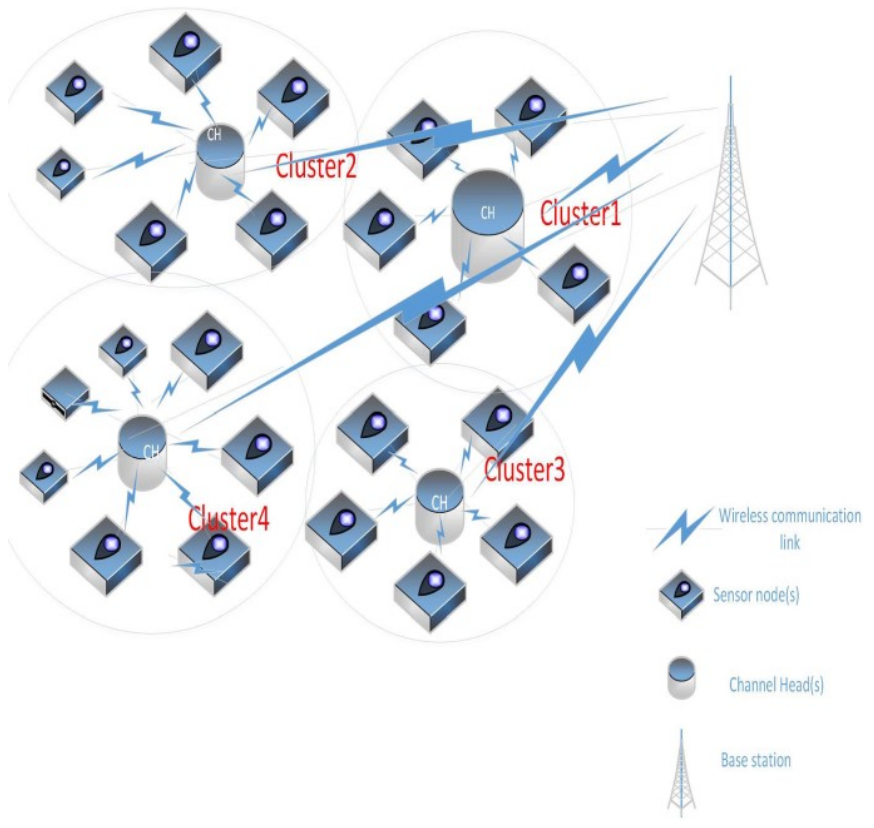




\section{S. Ajah et al.}

Figure 4 Multi-hop WSN structure (see online version for colours)

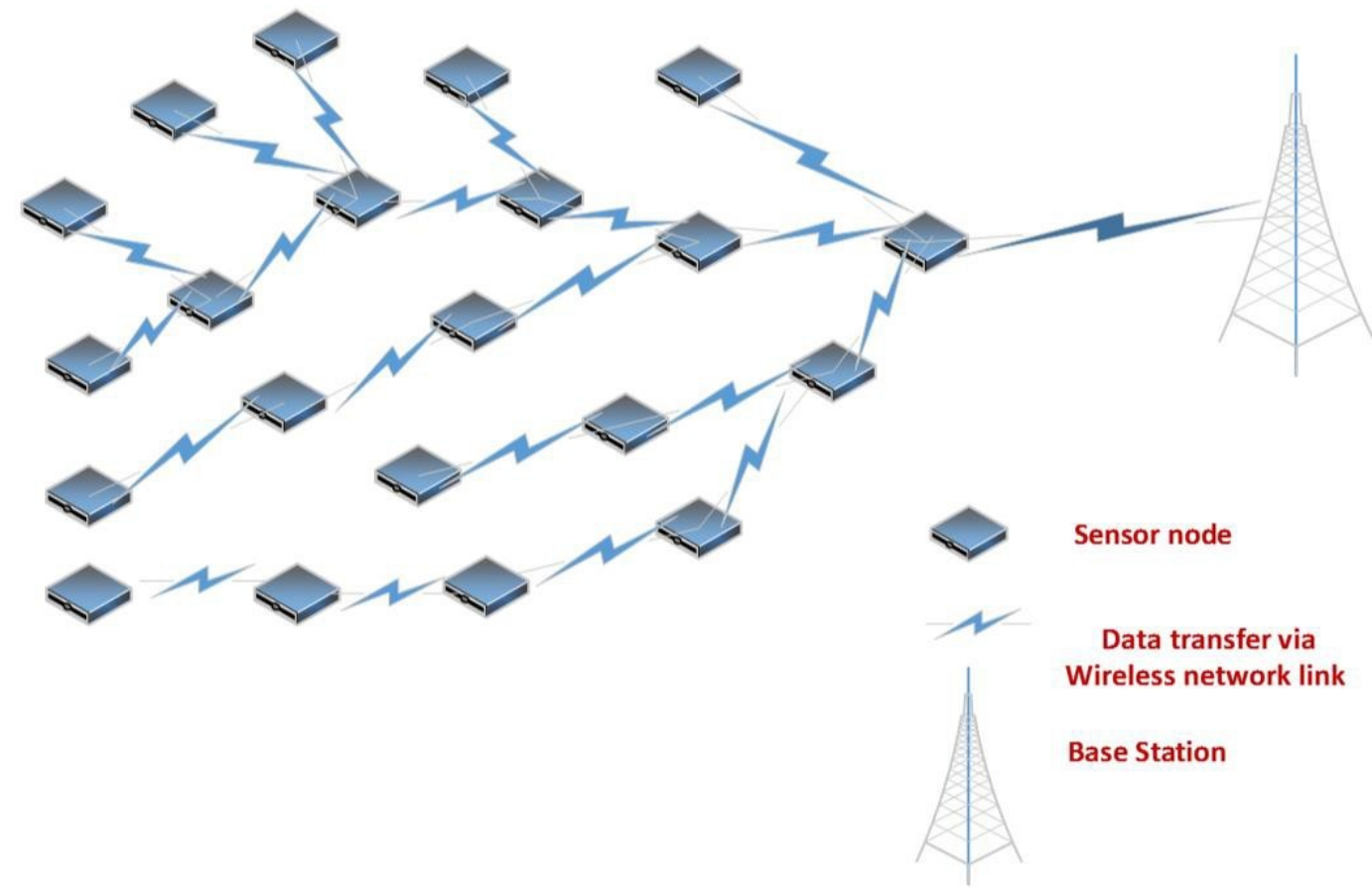

Structure with $\mathrm{CH}$ rotation among the nodes, but, the algorithm may not be efficient for some applications because of latency and communication overheads.

\subsubsection{Mobile sink-based WSN structure}

This is a new WSN communications paradigm that is growing in popularity in today's WSN. This mechanism will make the mobile sinks move randomly close to the nodes to collect the sensed data and then forward the collected data to the BS. This technique can be said to have more implementation complexity compared to aforementioned techniques but also the signalling energy cost is high.

On the aspect of the energy efficiency of the nodes, different techniques have being proposed by researchers such as Huang and Liu (2013), who proposed High Reliability Data Gathering Using Mobile Sink (HRDG-MS) in which the mobile sink moves randomly in WSN and periodically sends BEACON packets announcing its location to the neighbouring sensors. They observed that the existing proposed protocols based on the mobile sink mechanism as the means of enhancing the energy efficiency of WSN can be grouped into three: one-hop base strategy - this entails the mobile sink moves randomly in the network or in a planned direction and collects data from nodes via a one-hop strategy when the sinks are in their communications range, the other which this section is concerned is the clustering mechanism in which the CHs compress and send their collected data from their members to the mobile sink whenever in their range. The last is multi-hop base strategy in which sensors send their data to the mobile sink via multi-hop while the sink is mobile. Another approach is to use a planned adjustive data gathering algorithm for mobile sinks in large-scale WSNs to balance the power consumption of the sensors in the said network which will prolong the network lifespan (Saad et al., 2008). It can be argued that the latter proposal based on a mobile sink uses relatively more energy than HRDG-MS because of the implementation of clusters. The communications between the mobile sink and the nodes is only via the $\mathrm{CH}$ that collects the sensed data together before forwarding it to the mobile sink. The challenge of this model is their inabilities to incorporate rotation of $\mathrm{CH}$ which will make the $\mathrm{CHs}$ die off more quickly than other nodes as a result of high processing and communications energy demands.

\subsection{Gateway-based M2M structure}

This is one of the most popular M2M structures, in which 3GPP is proposed. The implementation of M2M applications using the current Mobile Cellular Network (MCN) has brought the idea of an intermediate gateway base M2M structure. This is to enable the MCN to be able to accommodate numerous $\mathrm{M} 2 \mathrm{M}$ applications and devices without affecting the Quality of Service (QoS) of the primary users. Laya et al. (2013) suggested that the M2M devices organise themselves locally with the aid of shortrange radio technologies such as Body Area Networks (BAN), Wi-Fi and WSN, creating an M2M area network. The M2M area network will connect to the core cellular network via M2M gateways which are equipped with a SIM card. Many papers have been written on enhancing the efficiencies of this structure to be able to accommodate numerous $\mathrm{M} 2 \mathrm{M}$ devices, to be energy efficient and maintain the security of the structure. 
Figure 5 Mobile sink WSN structure (see online version for colours)

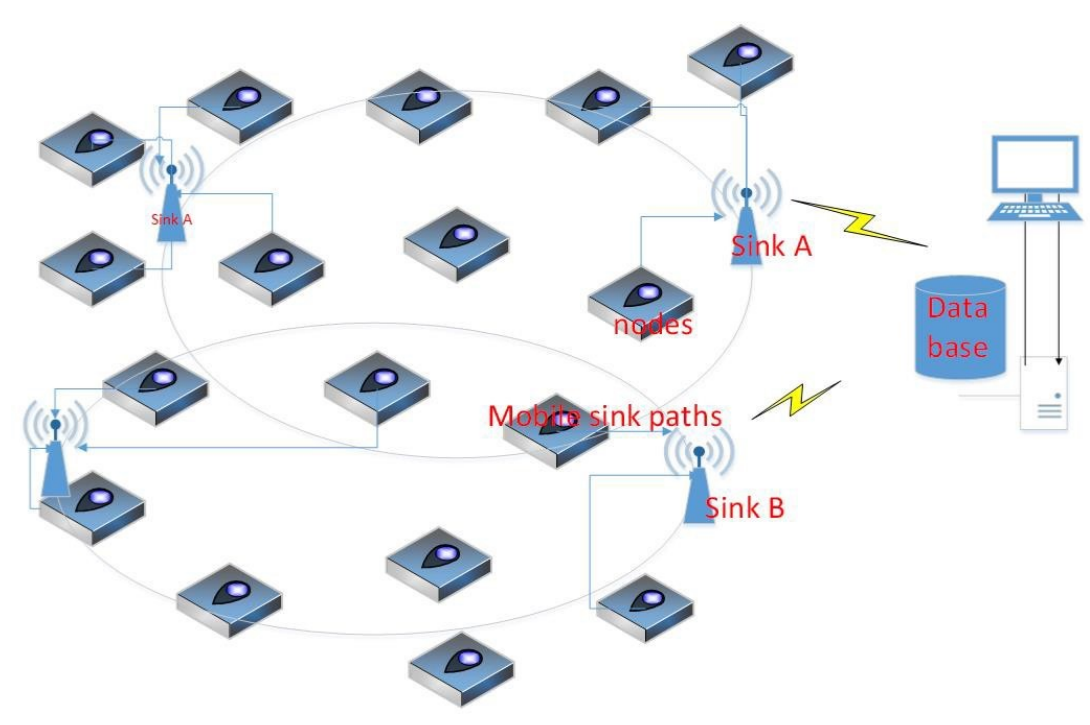

Based on 3GPP LTE-advanced, M2M devices will communicate to the LTE network directly if they are isolated or will communicate via a gateway (modem) if they are close to each other (Saedy and Mojtahed, 2011). The gateways have the capacity to communicate with the M2M devices and the cellular network. In relation to above mentioned, 3GPP also proposed Home NobeB technology, in which the connected home network will connect to the $3 \mathrm{G}$ network via one gateway (3GPP, 2008; 3GPP, 2009). Following this, Yunge and Zhipeng (2009) proposed a policy-based home NodeB gateway self-configuring structure which aimed to enhance the QoS of the said model in which the home NodeB establishes the link before transmission by sending a request to the policy server first, which aids to enhance the QoS. In addition, Kim and Choi (2010) proposed the effective M2M gateway selection algorithms for a geographical region-based query in which the registered $\mathrm{M} 2 \mathrm{M}$ devices can effectively select the appropriately registered M2M gateways in the M2M platform based on the locations of the devices and the gateway. While other authors (Luo et al., 2011) advocate for a multi-access gateway that can connect the heterogeneous network and is also capable of receiving and processing diverse sensed data especially for smart homes.

\subsection{Cellular-based M2M structure}

The cellular-based M2M structure is one of the most popular M2M standardisation activities going on today with so many already deployed by the mobile network operators and other M2M-based companies (Meters and More Open Technologies, 2012; Sierra Wireless, 2012; Sierra Wireless, 2013; Rogers, 2014). The popularity of the cellular-based $\mathrm{M} 2 \mathrm{M}$ structure is necessitated by the ubiquitous coverage, plug-and-play support and mobility support of the current MCN. Despite these merits, there are a number of major challenges of $\mathrm{M} 2 \mathrm{M}$ deployment in the current cellular network. The first is the management of the signals that will emanate from numerous M2M devices to avoid congestion of the network and the second is the adoption of energy sucking algorithms that are used for Human-to-Human (H2H) communications into an energy efficient algorithm that will be suitable for anticipated modus of operandi of M2M communications. 3GPP and IEEE standardisation associations are the popular bodies that are developing M2M standards based the existing cellular wireless network. While 3GPP is working on adjusting the air interface and the radio access of CDMA-2000 and LTE-advanced for M2M standards of which they have published their recommended standards based on the radio networks, IEEE 802.16p is working on doing the same on IMT-2000, which is also a cellular-based architecture (3GPP, 2008; 3GPP, 2009).

The main limitations of using MCN for M2M communications are the energy inefficiency of the existing $\mathrm{MCN}$ algorithms and the anticipated congestion of the network. It has been observed (Dhillon et al., 2013) that MCN including LTE was not designed for the link budget requirement of an $\mathrm{M} 2 \mathrm{M}$ traffic pattern. The need to tackle the congestions in $\mathrm{MCN}$ leads to the introduction of gateways which was discussed earlier. Another group that is working on M2M cellular-like communications paradigm based on lower frequencies spectra than the MCN spectra called Weightless SIG as discussed in the earlier sections (Weightless, 2013).

\subsection{Hybrid-based M2M structure}

This structure unites the cluster, gateway and cellular-based M2M structures. This structure can be seen as the potential standardised M2M structure because of the divergent M2M applications and standardisation activities. The rationale behind the hybrid M2M structure is that direct connections of all the M2M devices into MCNs may not be possible because of the large number of the devices to be deployed into the network, and also to harvest the merits associated with different M2M structures. Laya et al. (2013) observed that the random access channels of LTE and LTE-A networks are prone to congestions in M2M cases. Hence the 
need to integrate the short-range radio services and the wide area cellular networks, which will exploit the strengths of both types of technology to enhance the efficiency of M2M communications. Many experts in the field are proposing the use of wireless Local Area Networks (LAN) for connection of M2M devices in the same locality, while the applications that need global identity will be connected by amalgamating the gateways and the cellular network. One typical example of a hybrid M2M structure is the integration of the Home Area Network (HAN) and the Wide Area Network (WAN). The HAN devices can be connected by IEEE 802.15.4 standards like 6LoWPAN and ZigBee, which are energy efficient wireless standards in relation to most other M2M standards such as Wi-Fi, mash radios and connect to the WAN such as a Weightless and $\mathrm{MCN}$ via the gateway to the server.

\section{Energy implications of the standardisation activities}

Energy efficiency of M2M devices is one of the major design goals of the M2M networks and standardisations, based on the anticipated billions of devices expected into the networks in the nearest future (Ericsson, 2011). Consequently, the SDOs have recognised that a standard based on energy efficiency is the only way to actualise the dream of the connected world. In this section, we looked at the energy implications of different M2M specifications and different energy efficient algorithms being proposed by the SDOs and researchers based on each specification as the means of actualising an energy efficient connected world. Table 1 gives the properties of some of the SDOs.
From Figure 6, it can be seen that SDOs have different lifespan for their M2M devices even though some of them use similar frequency bands for communications. The SDO with the longest battery lifespan is the weightless specification, which uses white space for M2M communications, giving battery lifespan of over 10 years (Weightless, 2013). DASH7 Alliance uses a $433.95 \mathrm{MHz}$ spectrum for the M2M communications and has an expected lifespan of four years (Mohammad et al., 2012). The next SDO with long battery lifespan for their devices is the ZigBee specification, which has a lifespan of two years (Zigbee, 2014). Other SDOs that are adopting 3GPP M2M specifications have a battery lifespan of some days. The difference in the battery lifespan of SDOs even when using a similar spectrum is as a result of the difference in the communications principles and techniques they are using for M2M communications. The specification from weightless SIG seems to capture the ideal framework for M2M horizontal communications because of the vast spectrum they intend to use for M2M communications. The weightless specification has the longest lifespan as can be seen from Figure 6, and a maximum propagation range of $10 \mathrm{~km}$. The energy efficiency of the terminal is achieved through energy efficient algorithms, efficient coordinated sleeping and lowterminal transmission and reception energy (Weightless, 2013).

\subsection{Algorithms}

To achieve the require energy efficiency of M2M devices and networks, a holistic approach is required. To this end, this sub-section examines the energy efficiency principles being adopted by the SDOs in enhancing M2M energy efficiency.

Table 1 The SDOs and their properties

\begin{tabular}{|c|c|c|c|c|c|c|c|c|c|}
\hline $\begin{array}{c}\text { Standardisation } \\
\text { Bodies/ } \\
\text { Characteristics }\end{array}$ & 3 GPPS1 & $3 G P P 2$ & $I T U-T$ & $\begin{array}{c}\text { ETSI TC } \\
\text { M2M }\end{array}$ & $\begin{array}{c}\text { IEEE } 802.16^{\prime} S \\
M 2 M T G\end{array}$ & $\begin{array}{c}\text { IETF TASK } \\
\text { Group on } \\
\text { M2M/IoT }\end{array}$ & $\begin{array}{l}\text { ZigBee } \\
\text { Alliance }\end{array}$ & $\begin{array}{l}\text { DASH7 } \\
\text { Alliance }\end{array}$ & $\begin{array}{c}\text { Weightless } \\
\text { SIG }\end{array}$ \\
\hline Frequency (MHz) & $\begin{array}{r}800 \\
1800,2300, \\
2600,3600\end{array}$ & $\begin{array}{c}400, \\
800,1800, \\
2300,2600, \\
3600\end{array}$ & 1800 & Open & $2000-6000$ & $\begin{array}{c}868-868.6, \\
902-928, \\
2400-2483.5\end{array}$ & $\begin{array}{c}868 \\
915 \\
400-2483.5\end{array}$ & 433 & $470-790$ \\
\hline $\begin{array}{l}\text { Communications } \\
\text { Cost per device }(\$)\end{array}$ & $20+$ & $20+$ & - & $20+$ & $20+$ & $<10$ & $10+$ & $10+$ & $2+$ \\
\hline Max. Cell Range(KM) & $1+$ & $1+$ & $1+$ & - & $2+$ & $0.075+$ & $0.075+$ & 2 & 10 \\
\hline Indoor Characteristics & & & & - & Poor & Good & Good & Great & Excellent \\
\hline Modulations & $\begin{array}{r}\text { OFDMA/ } \\
\text { SC-FDMA }\end{array}$ & CDMA & - & - & OFDMA & QPSK & $\begin{array}{l}\text { BPSK, } \\
\text { OQPSK }\end{array}$ & GFSK & $\begin{array}{c}\text { Single-Carrier, } \\
\text { 16-QAM, } \\
\pi / 4-\mathrm{QAM}, \\
\pi / 2-\mathrm{BPSK}\end{array}$ \\
\hline Applications & Wide & Wide & Wide & Wide & Some & Some & Some & Some & Wide \\
\hline $\begin{array}{l}\text { Minimum device(s) } \\
\text { expected life Span } \\
\text { Maximum bit }\end{array}$ & Month & Month & & - & Month & 1 year & 2 years & 4 years & 10 years \\
\hline & 115,000 & 14,400 & - & - & $1 \mathrm{Gbps}$ & 250 & 250 & 200 & 16,000 \\
\hline
\end{tabular}


Figure 6 The spectrum and battery lifespan features of major SDOs (see online version for colours)

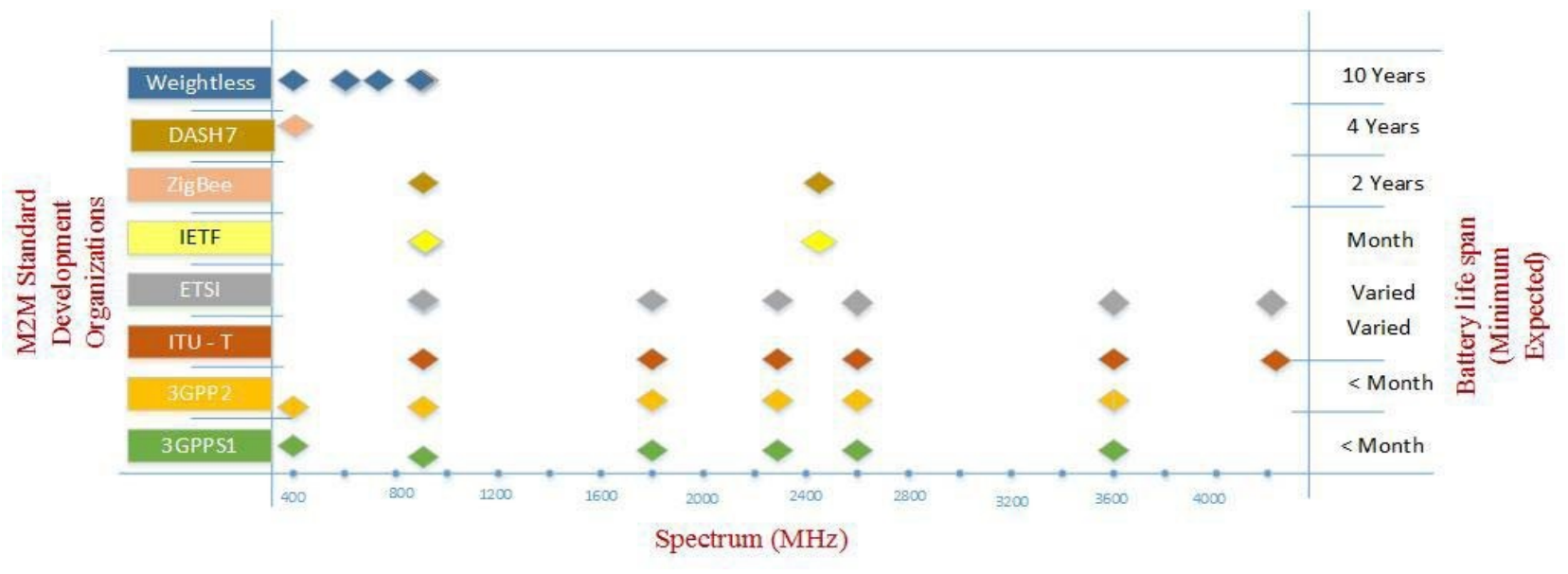

\subsubsection{Coordinated sleeping mechanism}

Coordinated sleeping and waking mechanisms have being described by researchers and experts as the techniques for enhancing the energy efficiency of wireless devices that are used for M2M communications, because of the planned traffics patterns associated with them (Wei and Shih, 2013). In the light of the above, 3GPPS1 have proposed discontinuous reception and transmission (DRX/DTX) in which the devices will turn off their radio interfaces and go into sleep mode whenever they are not communicating (3GPP TS $36.321,2012$ ). The weightless standard which is one of the SDOs with the longest lifespan for the devices also have a sleeping mechanism in place that will enable the devices to go to sleep for a predefined time and listens for a very short time as scheduled by the BS. Some of the devices that are connected to the mains will continuously sense and transmit their data to the BS (Weightless, 2013), while the ones with limited power will schedule a sense and transmit activity in a predefined time. This technique in conjunction with energy efficient techniques enables them to actualise over a decade lifespan for the devices. ZigBee, DASH7, IEEE 802.16's, IETF and other SDOs have also incorporated sleeping and waking mechanisms in their specification as the technique for elongating the lifespan of M2M devices (Maarten et al., 2013; National Instruments, 2013; Zigbee, 2014; Norair, 2009; Wei et al., 2010; Liu et al., 2013; Toscano and Lo Bello, 2012).

\subsubsection{Centralised data processing mechanism (cloud computing)}

This is another technique being employed by different SDOs in enhancing the energy efficiency of M2M networks devices. The sensors that are used for M2M communications only sense the data and transmit to the server, which then processes the data and issues a command based on the outcome of its processing to the node(s) for execution. The rationale behind the centralised data processing is to save the energy of the nodes that could have being spent processing the data to avoid associated complexity in the devices which would use the limited energy resource in maintaining the interfaces of their circuitry. Cloud computing can reduce the energy cost by $68-87 \%$ of office equipment like data servers (Google, 2012). In line with this discovery, some of the SDOs are looking into implementing cloud computing techniques in order to enhance the battery longevity of M2M devices. Also, it has been observed (Osawa, 2011) that cloud computing aids in the expansion of $\mathrm{M} 2 \mathrm{M}$ application areas by reducing the price and enhancing the performance of communications devices. In the light of the above, the Telecommunications Industrial Association (TIA), which is also one of the SDOs, have established a committee on cloud computing to amend their existing standard on M2M to be applicable to cloud services (TIA, 2011). In addition, ITU-T is looking at how to adjust the $\mathrm{M} 2 \mathrm{M}$ recommendations to access cloud computing services (ITU, 2013).

\subsubsection{Compressive Sensing (CS)}

This is another energy efficient technique that is being used in M2M communications. M2M communications generally consists more of data communications, in which the node(s) sense a signal and transmit via a wireless network to the server. The application of compressive sensing will reduce the quantity of data to be transmitted via the wireless network to the server. This technique is already being applied in M2M communications (EXALTED Consortium, 2012). Earlier work (Challa et al., 2002) on distributed data fusion using support vector machines is one of the fundamental principles of the compressive sensing technique. The data aggregation of M2M communications terminals can be done via the application of restricted isometric data gathering technique among the M2M devices, the architecture arranged data gathering technique, and the cluster arranged data gathering technique. Compressive sensing is then applied to the gathered data before transmission to the BS which then decompresses the data. The application of compressive sensing will reduce the amount of data that will be transmitted via the wireless network which will reduce the communications time, thereby enhancing the energy efficiency since it is the communications components of the wireless devices that consume over $50 \%$ of energy. 


\subsubsection{Green communications}

Following on from the negative environmental impact of energy inefficient devices, green communications is receiving high attention as the means for curbing the trends of high energy demands by the ICT sector. The report from Global e-sustainability Initiative (GeSI) indicated that green communications technique can aid in reducing the $\mathrm{CO}_{2}$ emission in the ICT sector by $15 \%$ by the year 2020 (GeSI, 2008). Also, according Amanna (2010), a medium size cellular network consumes as much energy as 170,000 homes and the energy cost of powering the BS accounts for over $50 \%$ of the services providers' overall expenses. The GeSI report and works of researchers, such as Miao et al. (2009), indicate that battery technology has not progressed as fast as the semiconductor technology. Consequently, the energy efficiency of the wireless devices needs a cross-layer approach to actualise (Miao et al., 2009). Some of the world organisations such as IEEE, EIT ICT Labs, Huawei and Green Communications have established technical working committees to oversee the implementation of green communications in ICT. With the recent events of M2M communications and numerous anticipated devices to be deployed into the network in the near future, green communications will be an efficient technique to actualise the energy efficient M2M communications.

\subsubsection{Cognitive Radio (CR)}

$\mathrm{CR}$ is a technique in wireless communications in which the transceiver is capable of intelligently detecting and using vacant channels for its transmission and reception while avoiding occupied channels. $\mathrm{CR}$ is necessitated by the spectra scarcity, according to Kaneko et al. (2008). CR is defined as the wireless communications technology in which the wireless devices communicate through an optimal wireless system based on the recognition of radio resources availability in heterogeneous wireless communications systems (Kaneko et al., 2008). The heterogeneous and number features of M2M communications have necessitated the application of these techniques to be able to achieve reliable and high energy efficiency of M2M communications system. Some of the SDOs are already using this technique, especially the ones whose specifications are based on the already occupied spectrum. The application of this technique in M2M communications will enhance the communications efficiency especially in the already occupied sub $1 \mathrm{GHz}$ spectrum, thereby reducing communications time and enhancing the energy efficiency.

\subsubsection{Artificial Intelligent (AI) and Swarm Intelligence (SI)}

This is another paradigm that is gaining popularity in $\mathrm{M} 2 \mathrm{M}$ communications because of its unique features. The autonomous nature of M2M communications necessitates the applications of AI and SI in order to enhance the reliability of the system. AI consists of tools, methods and systems for solving problems that will require the intelligence of human beings autonomously (Kasabov, 1996). While SI is an application of AI, it is an innovative distributed paradigm for solving optimisation problems whose concept comes from the biological like swarming, flocking and herding phenomena in vertebrates. SI is the quality of a system in which the collective behaviours of the naive agents that are interacting locally with their environment will lead to a coherent functional global pattern (Kumar and Thakur, 2012). While Bonabeau et al. (1999) define SI as the emergent collective intelligence of groups of simple agents. SI is a new paradigm, hence is being researched to improve the management and control of large number of interacting entities such as communications, computer and sensor networks.

\section{Open research topics}

- $\quad$ M2M standardisation based on sub $1 \mathrm{GHz}$ spectrum because of the cost, coverage and energy efficiency.

- The modalities for fast converging of the divergent $\mathrm{M} 2 \mathrm{M}$ standardisation activities into one.

- Enabling M2M servers in the cloud using cloud computing mechanism because of flexibility in connections and energy efficiency implications.

- Applications of CS on the horizontal M2M communications especially on the high-data-dependent applications.

- Enhancing the sensing efficiency of the M2M devices with sleeping mechanism.

- Efficient utilisation of CR techniques in enabling M2M communications in lower frequency spectrum.

- The application of SI technique in M2M communications.

\section{Conclusion}

This paper discussed different M2M communication standardisation activities by different global SDOs, the M2M structures followed the energy impact of different standardisation activities was discussed and analysed, and the energy efficient techniques being adopted by different SDOs in elongating lifespan of the devices was discussed. It is observed that weightless specification has the longest battery lifespan for M2M devices, cheapest in terms of cost per chip, the services cost, and infrastructure deployment cost and the range of the networks' signals per BS.

\section{References}

3GPP (2008) 3rd Generation Partnership Project; Technical Specification Group Radio Access Networks; $3 G$ Home NodeB Study Item Technical Report (Release 8), 3GPP.

3GPP (2009) Group Radio Access Networks; 3 G Home NodeB

Study Item, Technical Report (Release 8), 3GPP.

3GPP (2010) 3GPP TS 22.368 V10.1.0 (2010-06), 3GPP. 
3GPP (2011) 3GPP TS 22.368 V10.3.1 (2011-02), 3GPP.

3GPP (2012) 3GPP TS 22.368 V11.5.0 (2012-06), 3GPP.

3GPP (2013) 3GPP TS 23.682 V11.5.0 (2013-09), 3GPP.

3GPP TS 36.321 (2012) Evolved Universal Terrestrial Radio Access (E-UTRAN); Medium Access Control (MAC) Protocol Specification, 3GPP TS 36.321 Version 10.5.0 Release 10, ETSI, France.

3GPP2 (2010) S.R0141-0 V1.0: Machine-to-Machine Communication for cdna2000 Networks, 3GPP2.

6LowPAN (2014) 6LoWPAN Info, 07 February 2014. Available online at: www.6lowpan.org (accessed on 07 February 2014).

Amanna, A. (2010) Green Communications: Annotated Literature Review and Research Vision, Technical Report.

Anthony, L., Yee, L. and Martin, J. (2013) 'A Cellular-centric service architecture for machine-to-machine (M2M) communications', IEEE Wireless Communications, Vol. 20, No. 5, pp.143-151.

Beecham Research (2012) What M2M Users Really Want from M2M solutions: Results from Survey, Beecham Research, Cambridge.

Beecham Research Limited (2009) 'M2M world of connected services-Beecham.ppt, Community for Machine-To-Machine, Embedded Wireless, and Connected Consumer Devices Development, 5 November, p.1.

Bonabeau, E., Dorigo, M. and Theraulaz, G. (1999) Swarm Intelligence: From Natural to Artificial Systems, Oxford University Press, Oxford.

Challa, S., Palaniswami, M. and Shilton, A. (2002) 'Distributed data fusion using support vector machine', Proceedings of the 5th International Conference on Information Fusion, 8-11 July, pp.881-885.

Chen, Y., Zhang, S., Xu, S. and Li, G.Y. (2011) 'Fundamental trade-offs on green wireless networks', IEEE Communication Magazine on Energy Efficiency in Communications, Vol. 49, No. 6, pp.30-37.

DASH7 Alliance (2011) Automation \& Smart Energy (BASE) Overview, DASH7 Alliance.

Dhillon, H.S., Huang, H.C., Viswanathan, H. and Valenzuela, R.A. (2013) 'Power-efficient system design for cellular-based machine-to-machine communications', IEEE Transactions on Wireless Communications, pp.5740-5753.

Ericsson (2011) More than 50 Billion Connected Devices, Ericsson.

ETSI (2009) eHealth; Architecture; Analysis of User Service Models, Technologies and Applications Supporting e-health (ETSI TR 769 V1.1.1 (2009-02)), ETSI.

ETSI (2011a) Machine-to-Machine Communications (M2M); Smart Metering Use Cases (ETSI TR 102691 V1.1.1 (201005), ESTI.

ETSI (2011b) ETSI TS 102690 V1.1.1 (2011-10): Machine to Machine Communications (M2M); Functional Architecture, ETSI.

ETSI (2011) ETSI TS 122368 V10.5.0 (2011-07), 3GPP.

ETSI (2013a) Machine to Machine Communications (M2M); Use cases of Automotive Applications in M2M capable networks (ETSI TR 102898 V1.1.1 (2013-04)), ETSI.

ETSI (2013b) Machine-to-Machine Communications (M2M); Use Cases of M2M applications for Connected Consumer (ETSI TR 102857 v1.1.1 (2013-08)), ETSI.

EXALTED Consortium (2012) EXALTD WP4 - D4.4: Traffic Aggregation, EXALTED Consortium.
GeSI (2008) SMART 2020: Enabling the Low Carbon Economy in the Information Age, GeSI.

Global Standardization Committee (GSC) (2013) GSC MSTF Preliminary List of Global Organizations, Groups, Associations, FORA; and Other Entities with a Direct or Indirect Interest in Machine-to-Machine Standardization, GSC Standardization Task Force for GSC.

Google (2012) Google Apps: Energy Efficient in the Cloud, Google.

Heinzelman, W.B., Chandrakasan, A.P. and Balakrishnan, H. (2002) 'An application-specific protocol architecture for wireless microsensor networks', IEEE Transactions on Wireless Communications, Vol. 1, No. 4, pp.660-670.

Hersent, O., Boswarthick, D. and Elloumi, O. (2011) The Internet of Things: Key Applications and Protocols, John Wiley \& Sons LTD Publication, Chichester, UK.

Huang, J. and Liu, D. (2013) 'A high-reliability data gathering protocol based on mobile sinks for wireless sensor network', 22nd Wireless and Optical Communication Conference (WOCC), 16-18 May, pp.304-308.

IEEE Standards Association (2012a) IEEE Standard for Air Interface for Broadband Wireless Access Systems Amendment 1: Enhancement to Support Machine-to-Machine Applications, IEEE Standards.

IEEE Standards Association (2012b) IEEE Standard for Local and Metropolitan area networks - Part 15.4: Low-Rate Wireless Personal Area Networks (LR-WPANS), Amendment 2: Active Radio Frequency Identification (RFID) System Physical Layer (PHY). IEEE Std 802.15.4f, IEEE, New York.

ITU (2013) New ITU Standard on Cloud Computing Security and digital Object Architecture, 26 September. Available online at: www.itu.int/ITU-T/newslog/New+ITU+Standards+ On+ Cloud+Computing + Security + And + Object + Architecture.aspx (accessed on 21 February 2014).

ITU [182] (2011) Establishment of Internet of Things-GSI (IoTGSI) and first IoT-GSI Event, Geneva, 9-13 May to 05 April 2011. Available online at: www.itu.int/md/T09-TSB-CIR0182/en (accessed on 20 January 2014).

Kaneko, S., Nomoto, S., Ueda, T., Nomura, S. and Takeuchi, K. (2008) 'Predicting radio resource availability in cognitive radio - an experiment examination', 3rd International Conference on Communications (CrownCom'2008), 15-17 May, pp.1-6.

Kasabov, N.K (1996) Foundations of Neural Networks, Fuzzy Systems and Knowledge Engineering, Massachusetts Institute of Technology, MA, USA.

Kim, J., Lee, J., Kim, J. and Yun, J. (2013) 'M2M service platforms: survey, issues, and enabling technologies', IEEE Communications Surveys \& Tutorials, Vol. 16, No. 1, pp.61-76.

Kim, M. and Choi, S. (2010) 'Effective M2M gateway selection algorithms for geographical region-based query', Information and Communication Technology Convergence (ICTC), 1719 November, pp.413-414.

Ko, J.G., Terzis, A., Dawson-Haggerty, S., Culler, D.E., Hui, J.W. and Levis, P. (2011) 'Connecting low-power and lossy networks to the internet', IEEE Communications Magazine, Vol. 49, No. 4, pp.96-101.

Kumar, K. and Thakur, G.S.M. (2012) 'Advance applications of neural networks and artificial intelligence: a review', International Journal on Information Technology and Computer Science, Vol. 6, pp.57-68.

Laya, A., Alonso, L. and Alonso-Zarate, J. (2013) 'Is the random access channel of LTE and LTE-A suitable for M2M communications? A survey of alternatives', IEEE Communications Survey and Tutorials, pp.1-13. 
Levis, P., Tavakoli, A. and Dawson-Haggerty, S. (2009) Overview of Existing Routing Protocols for Low Power and Lossy Networks, IETF, California.

Lindsey, S. and Raghavendra, C.S. (2002) 'PEGASIS: powerefficient gathering in sensor information system', IEEE Aerospace Conference Proceedings, Vol. 3, pp.1125-1130.

Liu, Y., Yuen, C., Chen, J. and Cao, X. (2013) 'A scalable hybrid MAC protocol formassive M2Mnetworks', IEEE WCNC, pp.1-6.

Luo, H., Chang, C. and Sun, Y. (2011) 'Advanced sensor gateway based on FPGA for wireless multimedia sensor networks', International Conference on Electric Information and Control Engineering (ICEICE), 15-17 April, pp.1141-1146.

Maarten, W., Glenn, E., Luc, W., Charles, V. and Peter, H. (2013) 'Survey of the DASH7 alliance protocol for $433 \mathrm{MHz}$ wireless sensor communications', International Journal of Distributed Sensor Networks, pp.1-9.

Meters and More Open Technologies (2012) Endesa Leads Remote Management in Spain with 3.5 Million Smart Meters Installed. Available online at: www.metersandmore.com/ 2014/ 01/07/ endesa-leads-remote-management-in-spain-with-3.5-millionsmart-meters-installed (accessed on 10 February 2014).

Miao, G., Himayat, N., Li, Y. and Swami, A. (2009) 'Cross-layer optimization for energy-efficient wireless communications: a survey', Wireless Communications and Mobile Computing, Vol. 9, No. 4, pp.529-542.

Mohammad, A., Ahmad, U. and Varum, K.V. (2012) 'Dash7: performance', IOSR Journal of Electronics and Communication Engineering (IOSRJECE), Vol. 2, No. 5, pp.8-11.

Muruganathan, S.D., Ma, D.C.F., Bhasin, R.I. and Fapojuwo, A. (2005) 'A centralized energy-efficient routing protocol for wireless sensor network', IEEE Communication Magazines, Vol. 43, No. 3, pp.S8-13.

National Instruments (2013) Zigbee, National Instruments.

Norair, J.P. (2009) 'Introduction to DASH7 technology', Dash7 MenberClicks, pp.1-22.

Osawa, T. (2011) 'Practice of M2M connecting real-world things with cloud computing', FUJITSU Scientific \& Technical Journal, Vol. 47, No. 4, pp.401-407.

Rogers (2014) At-A-Glance, 23 April. Available online at: www.rogers.com/web/Rogers.portal? nfpb=true\&_windowla bel=investor_1_1\&ivestor_1_1_actionOverride $=\% 2$ Fportlets $\% 2$ Fconsumer\%2Finvestor\%2FshowGenericflexibleZoneActi on\&investor_1_1subAction=showGlanceWirelessAction\&_p ageLabel=IR_LANDING (accessed on 10 February 2014).

Saad, E.M., Awadalla, M.H. and Darwish, R.R. (2008) 'A data gathering algorithm for a mobile sink in large-scale sensor networks', 4th International Conference on Wireless and Mobile Communications (ICWMC'08), 27 July-1 August, pp.207-213.

Saedy, M. and Mojtahed, V. (2011) 'Ad hoc M2M communications and security based on 4G cellular systems', Wireless Telecommunications Symposium (WTS), 13-15 April, pp.1-5.

Sam, C. (2010) DASH7: Sensing on the Move, 14 August. Available online at: www.dailywireless.org/2010/08/14/ dash7-sensing-on-the-move/ (accessed on 20 January 2014).
Sierra Wireless (2012) Energy Solutions: Solutions for a Connected Energy Industry, Sierra Wireless, BC, Canada.

Sierra Wireless (2013) Advance Tracking Technologies, Inc [ATTI], Sierra Wireless AirPrime, BC, Canada.

TIA (2011) White Paper on Cloud Computing, TIA.

Toscano, E. and Lo Bello, L. (2012) 'Comparative assessments of IEEE 802.15.4/ZigBee and 6LoWPAN for low-power industrial WSNs in realistic scenarios', 9th IEEE International Workshop on Factory Communication Systems (WFCS), 21-24 May, pp.115-124.

Tsai, S-Y, Sou, S-I. and Tsai, M-H. (2012) 'Effect of data aggregation in M2M networks', 15th International Symposium on Wireless Personal Multimedia Communications (WPMC), 24-27 September, pp.95-99.

Usman, A. and Shami, S.H. (2012) 'Evolution of communication technologies for smart grid applications', Renewable and Sustainable Energy Reviews, pp.191-199.

Wan, J., Chen, M., Xia, F., Li, D. and Zhou, K. (2013) 'From machine-to-machine communications towards cyber-physical systems', ComSIS, Vol. 10, No. 3, pp.1105-1128.

Webb, W. (2012) Understanding Weightless: Technology, Equipment, and Network Deployment for M2M Communications in White Space, Cambridge University Press, Cambridge.

Wei, H-Y. and Shih, M-J. (2013) DeepSleep: IEEE 802.11 Enhancements for Energy-Harvesting Machine-To-Machine Communications, Intel-NTU Connected Context Computing Center Newsletter, National Taiwan University, Taiwan.

Wei, H-Y., Chou, C-C. and Zheng, Y-X. (2010) Reliable and Efficient Transmission to M2M Device Groups, IEEE C802.16p-10/0002r1.

Weightless (2013) Weightless Core Specification V1.0, Weightless SIG, Cambridge.

WIMAX Forum (2014) Technical Specifications, 06 Feb 20012014. Available online at: www.wimaxforum.org/resources/ technical-specifications (accessed on 06 February 2014).

Wu, J., Rangan, S. and Zhang, H. (2013) Green Communications, Theoretical Fundamentals, Algorithms and Applications, CRC Press, Boca Raton, FL.

$\mathrm{Wu}, \mathrm{X}$. and Wang, S. (2010) 'Performance comparison of LEACH and LEAC-C protocols by NS2', 9th International Symposium on Distributed Computing and Applications to Business, Engineering and Science (DCABES), 10-12 August, pp.254-258.

Yunge, L. and Zhipeng, G. (2009) 'Policy based home NodeB gateway self-configured structure', International Conference on Research Challenges in Computer Sciences (ICRCCS), 28-29 December, pp.116-119.

ZigBee (2010) Telecom Applications Profile Specification, ZigBee.

Zigbee (2014) ZigBee Specification FAQ, 20 February. Available online at: www.zigbee.org/specifications/ZigBee/FAQ.aspx (accessed on 20 February 2014).

ZigBee Alliance (2010) ZigBee Remote Control Public Application Profile Specification Version 1.1.0, Zigbee Alliance.

ZigBee Alliance (2012) 'ZigBee alliance celebrate 10 years: delivering the internet of things and energy management and efficiency', ZigBee Alliance Press Release, pp.1-3. 